\title{
El efecto de la estructura social sobre el gasto en atención a la discapacidad
}

\author{
Ángel Belzunegui Eraso \\ Xavier Puig Andreu \\ Francesc Valls Fonayet \\ Universitat Rovira i Virgili. Cátedra de Inclusión Social \\ cinclusiosocial@urv.cat; xavier.puig@urv.cat; francesc.valls@urv.cat
}

\begin{abstract}
Resumen: Este artículo examina el gasto que los hogares españoles con personas con discapacidad realizan para atender la discapacidad a partir de la información extraída de la Encuesta de Discapacidades, Autonomía y Dependencia del año 2008, última edición disponible. Se constata una importante diferenciación social dentro del colectivo de personas con discapacidad, según la cual a mejor posicionamiento social, mayor gasto en discapacidad, sea cual sea el grado de limitación. De igual forma, se analiza la elasticidad de dicho gasto, y se realiza una estimación del peso «social» que supone.

Palabras clave: discapacidad, estructura social, pobreza, gasto social.
\end{abstract}

The effect of social structure on disability support spending

Abstract: This article examines the expenditure incurred by Spanish households in order to be able to care for disabled family members. Data is taken from the Spanish Survey on Disabilities, Autonomy and Dependence for the year 2008, which is the latest data available. There is significant social differentiation among disabled people in that better-off individuals spend more resources on dealing with disability regardless of the degree of limitation. Likewise, we analyze the elasticity of the expenditure and estimate its «social» weight.

Keywords: disability, social structure, poverty, social expenditure. 


\section{Introducción}

Abundan los estudios que ponen de relieve la diferenciación social del colectivo de personas con discapacidad con respecto al resto de colectivos sociales, así como la fuerza que la discriminación social tiene para convertir la discapacidad en un eje de desigualdad y exclusión social. Aquí nos proponemos estudiar la diferenciación social en el seno mismo del amplio y variado colectivo, es decir, la estructura social de la discapacidad. ¿Existen diferencias reseñables en el gasto de atención a la discapacidad en función de la posición social de los hogares?

Para tal fin explotaremos la información de la Encuesta de Discapacidades, Autonomía y Dependencia para hogares en su última versión disponible, del año 2008 (EDAD 2008, en adelante). Esto significa que, por un lado, nos será posible trabajar con variables directas acerca de la discapacidad de las personas, y no con las habituales variables indirectas propias de las encuestas que todavía no incorporan la discapacidad como una de las variables indispensables, como sí son el sexo, la edad o los estudios. Por el otro, significa que nos será posible llevar a cabo un estudio sobre el gasto en discapacidad, un indicador que, a diferencia de la renta, nos permite examinar los recursos de salida (más que los de entrada) o, si se prefiere, la renta actual, la renta acumulada y la renta esperada.

La EDAD 2008, en su versión de hogares, parte de una potente muestra de 96.000 hogares (alrededor de 260.000 personas), en un 5,6\% de los cuales vive alguna persona con discapacidad y con un gasto explícitamente destinado a la atención de dicha discapacidad. Esta es la submuestra para el presente trabajo, sobre la cual se han aplicado distintas técnicas de análisis estadístico con la finalidad de comparar el gasto entre las distintas posiciones sociales de los hogares, tales como un análisis de correspondencias múltiple y un análisis de clasificación biepática. Para la posición social del hogar se ha tomado la referencia del nivel de estudios, la posición laboral y el nivel salarial de la persona con una aportación mayor de ingresos al hogar.

Estructuraremos este artículo de forma que en primer lugar se expongan el marco conceptual en el que se inscribe el planteamiento y las hipótesis que nos guían en el análisis; en segundo lugar expondremos los resultados generales del gasto en discapacidad en España; en tercer lugar abordaremos el gasto social de la discapacidad en España, relacionando el gasto con las variables estructurales elegidas; en cuarto lugar mostraremos una estimación del peso del gasto «social» en función de la gravedad de la limitación; y finalmente expondremos las conclusiones extraídas. 


\section{Planteamiento conceptual e hipótesis}

La noción de discapacidad es problemática, escurridiza y difícil de atrapar. Por un lado, tiene significaciones distintas en función del contexto de utilización y de la perspectiva teórica de la que se parta (Hughes y Paterson, 1997; Michailakis, 2003; Vehmas y Taylor, 2004; Amiralian et alii, 2000; Gerschick, 2000; Gustavsson, 2004). Por el otro, rivaliza con otros términos para describir - y construiruna determinada realidad, como pueden ser la deficiencia, en su vertiente más fisiológica y médica, y la diversidad funcional, en su vertiente más social y emancipadora (Verdugo, 2003; Egea y Sarabia, 2004, Díaz, 2009; Rossignol, 1999; 2006). Sin embargo, tras la superación de etiquetas extremadamente denigrantes como «idiota», «imbécil», «cretino» o «subnormal» (Egea y Sarabia, 2001:17), o como minusválido o disminuido, más recientemente, el término persona con discapacidad se erige como la categoría más ampliamente aceptada y utilizada por la comunidad científica. Esta categoría nos informa de algún tipo de limitación en las actividades de la persona, si no desde un punto de vista más neutro, sí sin menoscabo del valor de la persona en su conjunto. Por esta razón se utilizará, en este trabajo, el término de persona con discapacidad.

Con la finalidad de delimitar la idea de discapacidad y unificar criterios, la Organización Mundial de la Salud (OMS, 2001) elaboró la Clasificación Internacional del Funcionamiento, de la Discapacidad y de la Salud (CIF) el año 2001 (revisión de clasificaciones anteriores ${ }^{1}$ ). La CIF aborda la discapacidad con la intención de ser de utilidad para distintos posicionamientos teóricos, en especial para las perspectivas médica y social².

Sin embargo, la definición que la CIF propone no resuelve del todo el problema de la polisemia, dado que la OMS (2001:3) describe la discapacidad (en oposición a la noción de funcionamiento) como aquello que «engloba las deficiencias, limitaciones en la actividad o restricciones en la participación». A juicio de Egea y Sarabia (2001:24), se trata de un concepto que «se eleva a término 'paraguas' para todas las condiciones de salud negativas», pero que abarca tanto condiciones de salud como condiciones sociales: primero las deficiencias, que son problemas en las funciones y estructuras corporales; segundo, las limitaciones en la actividad, entendidas como las dificultades que el individuo pueda tener en el desempeño o realización de la misma; y tercero, las restricciones en la participación, es decir, los problemas que un individuo puede experimentar al involucrarse en situaciones vitales (Hemmingsson y Jonsson, 2005).

1 Para profundizar en la comparación entre las distintas clasificaciones de discapacidad de la OMS véase Egea y Sarabia (2004).

2 Una lectura crítica de la clasificación puede consultarse en Chapireau (2001). 
Puesto que nosotros, en este análisis, centraremos nuestra mirada en la información contenida en la EDAD 2008, compartiremos la delimitación que en dicha encuesta se realiza. A pesar de que la misma encuesta asegura estar «impregnada de la filosofía de la nueva Clasificación Internacional del Funcionamiento, de la Discapacidad y de la Salud» (INE, 2010:3), lo cierto es que sus bases conceptuales «apenas tienen en cuenta el nuevo sistema de la OMS para clasificar el funcionamiento y la discapacidad, sino que siguen ancladas en los planteamientos de la clasificación de 1980» (IOÉ, 2013:130). En efecto, el planteamiento de la EDAD 2008 dista del de la OMS en que se omiten «los factores sociales (ambientales y personales) implicados en el proceso, especialmente las estrategias adoptadas por el sujeto afectado, por su familia y demás agentes e instituciones para asumir y abordar la discapacidad», aunque cuenta con la ventaja de establecer una «clasificación objetiva y jerarquizada en el plano del individuo» (IOÉ, 2013:131).

La EDAD 2008 identifica el concepto de discapacidad con el de limitaciones importantes «para realizar las actividades de la vida diaria que hayan durado o se prevea que vayan a durar más de un año y tengan su origen en una deficiencia» (INE, 2010:4). Así pues, se establece como criterio que solo serán consideradas como discapacidad las limitaciones en las actividades cuyo grado de severidad sea importante, no meramente moderado o leve. Con respeto a las deficiencias, el estudio se limita asimismo a aquellas que han originado una limitación en la persona.

Una vez seleccionadas las personas que forman parte de nuestro análisis según las restricciones expuestas, nos centramos en el estudio de la relación entre estructura social y discapacidad. Este estudio lo abordamos por el lado del gasto; en concreto, nos centramos en el análisis del gasto de los hogares con personas con discapacidad. El debate sobre el uso de la variable de referencia en los estudios sobre pobreza nace con las primeras encuestas cuantitativas sobre presupuestos familiares y sobre condiciones de vida. Las investigaciones se han organizado en torno a dos enfoques predominantes en función de la variable de referencia utilizada: la renta o el gasto. En ambos enfoques, la estrategia metodológica equipara el nivel de bienestar de las personas con la disponibilidad material de recursos, ya sea calculando su volumen de entrada (renta) o de salida (gasto) durante un periodo concreto. Como pasa también alrededor de la definición de pobreza, el uso de un indicador $u$ otro no es un hecho banal ni neutro, puesto que los resultados variarán en función de la elección (De la Cal, 2009). Como ejemplo, Haveman y Bershadker (2001) proponen utilizar la incapacidad para ser autosuficientes como indicador de pobreza y desde una visión más multidimensional, Gordon 
(2005) propone incluir en el concepto de pobreza aspectos como el hambre, la malnutrición, la mala salud, el acceso a servicios básicos, la vivienda inadecuada o la falta de vivienda, los entornos inseguros, la discriminación social o la exclusión en la toma de decisiones o de la sociedad civil.

La variable renta proporciona una imagen real de la capacidad económica del hogar en un momento dado a pesar de que se limita a informar de la mera disponibilidad de recursos excluyendo el ahorro y el patrimonio. Por su parte, la variable gasto ofrece una mayor estabilidad ante los cambios puntuales de la capacidad económica del hogar, puesto que se basa en la idea de renta permanente, que incluye la renta actual pero también la renta acumulada en periodos anteriores y la renta esperada en el futuro. Así, en cuanto a la validez del constructo metodológico, el gasto es un indicador que se ajusta mejor que la renta a la realidad que se pretende estudiar (las condicionas de vida), y se adapta más bien a la idea de que el nivel de vida depende de la utilidad que las personas dan a sus recursos más que de su simple disponibilidad.

Sin embargo, trabajar con el gasto requiere algunas precauciones: en primer lugar, no siempre es correcto relacionar nivel de gasto y disponibilidad de medios (como la no correspondencia entre los niveles de renta de la población jubilada y la austeridad en el consumo de este colectivo); en segundo lugar, la inclusión de bienes duraderos puede alterar el volumen de gasto de un año a otro; $y$ en tercer lugar, la dificultad que tienen los hogares para realizar cálculos exactos en el gasto invita a los redondeos.

Mercader-Prats (1998) detecta que, aunque la correlación entre renta y gasto es esperable, no es inequívoca. Según sus cálculos, solo la mitad de la población definida como pobre por sus bajos niveles de renta pertenecería al grupo de población de bajo nivel de consumo, lo cual evidencia la influencia que tiene la elección metodológica de la variable de referencia sobre los resultados obtenidos (Pérez, 2009; Cantó et alii, 2000).

La operación estadística del Panel de Hogares de la Unión Europea (UE) entre 1994 y 2001 y de la Encuesta de Condicionas de Vida (desde 2004) prácticamente acabó con este debate, puesto que solo se aporta información sobre la renta de los hogares. Por esto, y por la mayor comparabilidad de esta variable con los datos europeos (a través de las encuestas estatales pertenecientes al modelo europeo armonizado EU-SILC), desde la segunda mitad de la década de 1990 el gasto ha pasado a ser una variable secundaria, si no olvidada, en la medida de la pobreza. Nosotros la hemos recuperado aquí para observar cómo se manifiesta en función de algunas variables que hemos asociado al empobrecimiento. 
Pero la literatura académica que aborda la discapacidad y la estructura social suele tener en la renta el indicador de referencia. Así ocurre en el caso de los trabajos que ponen de relieve la intensa asociación entre discapacidad y pobreza (IOÉ, 2003; Ferreira y Díaz, 2007; Barnes, 2010; Díaz, 2011), y que muestran la pobreza como una «característica persistente de la vida de las personas con discapacidad en todo el mundo» cuyo vínculo con la discapacidad «ha sido reconocido de manera inequívoca a nivel internacional» (Barnes, 2010: 8; ONU, 2008; Albert, 2005; Barnes y Mercer, 2005; Banco Mundial, 2007).

En efecto, esta prevalencia mayor de la discapacidad entre las familias pobres, según la cual a mayor nivel de renta menos personas con discapacidad, puede tener su explicación en varios factores entrelazados. De acuerdo con el Colectivo IOÉ (2003: 45-46), «En primer lugar, las personas en situación precaria suelen padecer unas condiciones de vida menos saludables y mayores riesgos de contraer enfermedades o sufrir accidentes, puntos de partida de las deficiencias y las discapacidades. En segundo lugar, las posibilidades de rehabilitación y reinserción de tales personas son menores, al no disponer de suficientes recursos económicos», además de tener en cuenta que «las discapacidades afectan más a personas ancianas, quienes frecuentemente disponen de menores niveles de renta» (Barrientos, Gorman, y Heslop, 2003; Minkler, Fuller-Thomson y Guralnik, 2006).

$\mathrm{Y}$ es que la discapacidad actúa tanto como variable independiente de la pobreza, puesto que condiciona en gran medida la posición del individuo en la estructura social, como de variable dependiente de ella, puesto que surge de las situaciones de vulnerabilidad social (Díaz, 2011).

En este trabajo lo que se pretende, no obstante, no es ahondar en la cuestión de la posición de los colectivos con discapacidad en la estructura del conjunto social, sino más bien analizar la estructura social del propio colectivo. Dicho de otro modo, ver las desigualdades existentes dentro del colectivo de hogares con alguna persona con discapacidad, teniendo en cuenta variables sociodemográficas como el nivel de estudios, la situación laboral y el nivel de ingresos. Por razones operativas, nos adaptaremos a la información facilitada por la EDAD 2008 y tomaremos como referencia la persona con mayor aportación de ingresos al hogar — sea hombre o mujer, tenga alguna discapacidad o no-.

Así que, a continuación, se plantean las hipótesis que ordenan y guían este trabajo de análisis de la estructura social de la discapacidad:

1. A mayor gravedad de la discapacidad, mayor importancia de la dimensión físico-medica en el gasto total.

2. A mayor nivel socioeconómico del hogar, mayor volumen de gasto para un nivel de limitación dado. Esta hipótesis se puede descomponer en las 
siguientes subhipótesis: 2.1. A mayor nivel de estudios, mayor gasto en discapacidad para un nivel de limitación dado. 2.2. A mejor posición laboral, mayor gasto en discapacidad para un nivel de limitación dado. 2.3. A mayor nivel de ingresos, mayor gasto en discapacidad para un nivel de limitación dado.

3. El peso de la dimensión social en el gasto por discapacidad aumenta a medida que disminuye la gravedad de la discapacidad. Los hogares con mejor situación socioeconómica dedican mayor volumen de gasto a la dimensión social de la discapacidad.

\section{El gasto en atención a la discapacidad en España}

La EDAD 2008 dispone de una serie de preguntas sobre el gasto que realizan los hogares para atender la discapacidad de alguno de sus miembros. Esta información no está exenta de dos problemas relevantes. El primero tiene que ver con lo que se ha comentado anteriormente, esto es, la dificultad de los hogares para calcular los costes anuales de la discapacidad, tanto en la cuantificación de las diversas partidas como en el discernimiento del grado en que los gastos son imputables a la discapacidad (exclusivamente, parcialmente, etc.). El segundo problema es la elevada falta de respuesta, que se traduce en que un $80,2 \%$ de los hogares no responde a esta variable. La práctica totalidad corresponde a hogares sin miembros con discapacidad y, por tanto, se les puede imputar un gasto inexistente, de forma que son casos que podemos desestimar. Aun así, un 1,7\% de hogares sin respuesta declara tener algún miembro con discapacidad. Por otro lado, el 14,2\% de los hogares tiene algún miembro con discapacidad, pero declaran no tener ningún gasto asociado a la discapacidad. Finalmente, el 5,6\% del total de los hogares españoles declara tener que hacer frente a algún tipo de gasto por motivos de discapacidad de alguno de sus miembros. Es en este porcentaje de hogares en el que hemos centrado el análisis de la cuantía y la naturaleza del gasto por discapacidad.

Este $5,6 \%$ de hogares españoles con algún miembro con discapacidad y con gasto declarado por discapacidad gasta una media de 2.787 euros anuales en bienes y servicios para atender la discapacidad. Aun así, esta cuantía se encuentra sensiblemente sesgada por la presencia de un pequeño porcentaje de hogares con un gasto muy elevado, superior a los 20.000 euros anuales. Otros indicadores menos sensibles a la presencia de casos extremos (como la mediana, la media recortada al $5 \%$ o varios estimadores de centralidad más robustos, como el estimador $\mathrm{M}$ de Huber) apuntan a una cuantía sensiblemente inferior, que oscila entre 1.100 y 2.000 euros anuales. 
Como se observa en el gráfico 1, el $50 \%$ de los hogares españoles presentan un gasto inferior a 1.100 euros anuales y un $25 \%$ gasta menos de 400 euros anuales. La parte superior de la distribución presenta mayor elasticidad: prácticamente un $25 \%$ de los hogares con personas con discapacidad dedican más de 3.000 euros anuales a la discapacidad, mientras que el $10 \%$ superior dedica más de 6.500 euros anuales.

Gráfico 1. Distribución acumulada de hogares en función del gasto dedicado a la discapacidad, en euros anuales (solo hogares con gasto dedicado a la discapacidad). España, 2008.

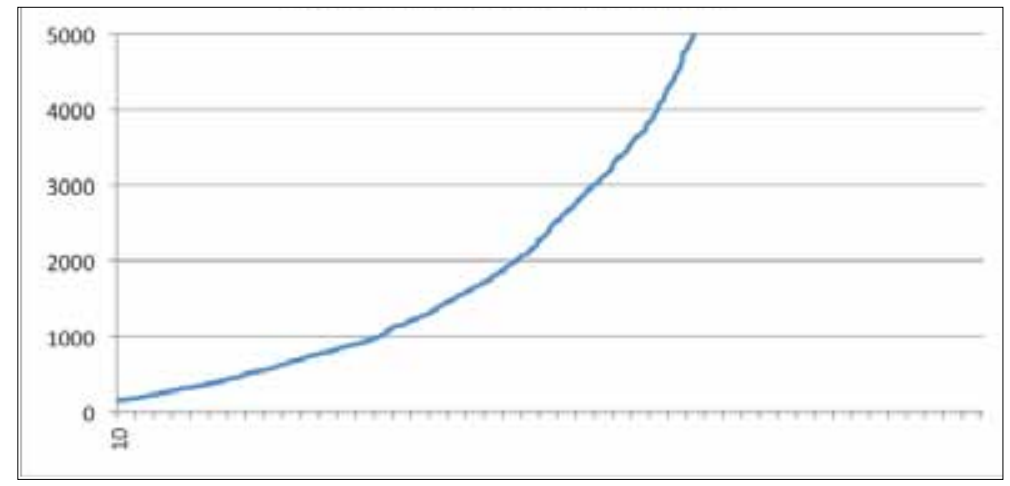

Fuente: elaboración propia a partir de la EDAD 2008.

Las condiciones de vida de los hogares con personas con discapacidad están determinadas por una doble circunstancia. La primera tiene que ver con el mayor gasto para lograr similares estándares de calidad de vida que el resto de hogares que no tienen personas con discapacidad, circunstancia que favorece un efecto unificador del conjunto de los hogares con miembros con discapacidad. Es decir, aquellos hogares tienen mayores necesidades (en términos de fármacos, de atención y cuidado personal, de capacitación de la persona, de adquisición de bienes y servicios adecuados, de adaptación de la vivienda, etc.). Y, por tanto, necesitan gastar más recursos para poder lograr niveles equivalentes de funcionalidad social. Esta idea parte de la teoría de capacidades de Sen $(1984,1995,1999)$, según la cual aquello relevante en el estudio de la pobreza no es la cantidad de recursos disponibles, variable que solo se mueve en un nivel instrumental, sino el nivel de vida de las personas, que es aquello intrínsecamente importante. La discapacidad o un mal estado de salud, entre otros factores, dificultan el acceso a los recursos, pero a la vez también impiden que un mismo volumen de recursos sea indicativo de un mismo nivel de vida, puesto que la capacidad de transformar estos recur- 
sos en funcionamiento social no es equivalente. Al respecto, estudios como el de Dobson, Middleton y Beardsworth (2001) han mostrado el sobrecoste que supone para las familias los gastos en educación de niños con discapacidad grave. Los autores presentan patrones de gasto real de las familias de 182 niños con discapacidades graves relacionándolos con la capacidad económica y de gasto de las propias familias. En la misma línea, Newacheck, Inkelas y Kim (2004) explican los patrones de gasto en los cuidados de salud de niños con discapacidad, poniendo de manifiesto las diferencias socioeconómicas en las familias con niños discapacitados.

En España, diferentes investigaciones han intentado cuantificar el volumen de recursos suplementarios que los hogares con personas con discapacidad tienen que alcanzar para conseguir un nivel de vida similar al de los hogares sin personas con discapacidad. En esta línea, Braña y Antón (2011) han cuantificado los costes de la discapacidad y sus efectos sobre la pobreza concluyendo que, una vez cuantificados estos costes (que suponen alrededor del $40 \%$ de la renta del hogar en casos de discapacidad moderada y del $70 \%$ en casos de discapacidad severa), la incidencia, la intensidad y la severidad de la pobreza aumentan de manera sustancial: si en el hogar habita un miembro con discapacidad moderada, el riesgo de pobreza se duplica (del 29,6\% al 59,3\%), mientras que en los hogares con miembros con discapacidad severa se triplica y pasa a incumbir a la práctica totalidad de hogares (del 30,3\% al 93\%). Un efecto similar sucede con las tasas de pobreza extrema: en los hogares con discapacidad moderada el porcentaje de hogares que caerían en la pobreza extrema sube del 3,5\% al 17,3\%, mientras que en los hogares con discapacidad severa lo hace del $2,8 \%$ al $56,4 \%{ }^{3}$.

Hay un segundo aspecto que está menos tratado, analíticamente hablando. Se aprecia una heterogeneidad interna del colectivo originada por la dimensión social de la discapacidad. Aunque se puede decir que se trata de un colectivo que soporta peores condiciones de vida que la población no discapacitada, no se debería homogeneizar a toda la población discapacitada, puesto que ella misma está estructurada (diferenciada socialmente) en base a factores de jerarquización social, como la clase, el género o la edad. Esta heterogeneidad queda patente al observar los comportamientos de gasto por discapacidad, cuyo análisis nos ocupará en los siguientes apartados.

\footnotetext{
3 En una línea similar, Malo y Dávila (2006), mediante una modificación en la ponderación de los miembros del hogar para dar un mayor peso a los miembros con discapacidad (asumiendo la hipótesis de que su presencia aumenta los costes del hogar en mayor medida que una persona sin discapacidad), calculan que el riesgo de pobreza aumentaría entre un $60 \%$ y un $80 \%$ en esta simulación, hasta duplicarse en el caso en que en el hogar residan dos o más personas con discapacidad.
} 


\section{La dimensión social del gasto en discapacidad}

Que la presencia de individuos con discapacidad en el hogar implica un aumento del gasto es un dato previsible, como lo es también que este aumento varía en función del tipo y del grado de discapacidad. Por ejemplo, sabemos que los hogares con personas con discapacidad que están gravemente limitadas dedican una media de 3.713 euros anuales a atender la discapacidad, un gasto entre un $36 \%$ y un $70 \%$ superior al de aquellos hogares en que la discapacidad, o bien no supone ninguna limitación, o en todo caso supone una limitación moderada.

Pero la discapacidad no solo se explica por medio de factores médicos o físicos. Las datos disponibles indican que una parte sustancial del efecto que la discapacidad tiene sobre las condicionas de vida de los hogares depende de factores sociales. Y en determinadas situaciones, la dimensión social de la discapacidad llega a ejercer un mayor peso explicativo que la estricta dimensión físico-médica.

Nos aproximamos a la cuestión mediante el análisis de la relación entre la posición social del hogar y el gasto dedicado a la discapacidad. Para evitar un análisis demasiado genérico sobre el efecto de la discapacidad en el coste anual, hemos segmentado la variable «limitación para hacer algunas actividades» en tres categorías y las hemos analizado de forma independiente (algún miembro del hogar se encuentra gravemente limitado; algún miembro del hogar se encuentra limitado, pero ninguno de ellos gravemente; y ningún miembro del hogar se encuentra limitado). Para definir la posición social del hogar se han recogido los datos relativos a la persona de referencia, definida como aquella que, residiendo habitualmente en el hogar, es la que más aporta periódicamente en términos monetarios a su presupuesto total.

Dos de los indicadores clave de la posición social son el nivel formativo logrado y la relación con el mercado de trabajo. En cuanto al primero, los hogares con miembros con discapacidad tienden a aumentar el gasto dedicado a la discapacidad a medida que aumenta el nivel formativo de la persona de referencia (hipótesis 2.1), con un aumento del gasto de entre un $22 \%$ y un $83 \%$ por cada etapa superior de nivel formativo, en función del grado de limitación de la persona discapacitada, y siempre en la línea según la cual a mayor grado de limitación, mayor gasto destinado a la discapacidad. Así, los hogares en que la persona de referencia tiene estudios universitarios son los que más invierten en atender la discapacidad, especialmente si la persona con discapacidad sufre una limitación grave: en este caso el gasto medio asciende hasta los 5.087 euros anuales, muy por encima del que dedican el resto de hogares: 3.421 euros en los hogares en los que la persona de referencia tiene estudios secundarios, 4.631 euros en los hogares con estudios primarios y 2.950 euros en los hogares en los que la persona de referencia no 
tiene estudios. Pero no únicamente: incluso los hogares encabezados por una persona de referencia con estudios universitarios y con una persona discapacitada sin limitación grave o que no sufre ninguna limitación, invierten más en atender la discapacidad que el resto de hogares en que la persona discapacitada sufre una limitación grave, solo a excepción de los hogares con estudios primarios (ligados a una edad avanzada). De aquí la necesidad de resaltar el componente social de la atención a la discapacidad, que va más allá de una lectura exclusivamente físicomédica.

Cuando la discapacidad no supone una limitación, o supone una limitación moderada, el gasto dedicado a su atención se relaja (hipótesis 1). Esta reducción presenta dos características relevantes. La primera tiene que ver con la relación establecida anteriormente: a una posición social de mayor bienestar le corresponde un mayor gasto en la atención a la discapacidad. La segunda es que la reducción también presenta un comportamiento desigual según la situación socioeconómica del hogar: los hogares con menor nivel formativo reducen sustancialmente el gasto dedicado a la discapacidad cuando esta no supone una limitación grave en relación a cuando sí que la supone, a diferencia de los hogares con mayor nivel formativo, con un comportamiento más estable entre los diferentes escenarios. La reducción es del 35-40\% del gasto en los hogares en los que la persona de referencia no tiene estudios, del 50-55\% cuando la persona de referencia tiene estudios primarios, de un 15-35\% cuando tiene estudios secundarios, y en torno al $18 \%$ cuando tiene estudios universitarios. Como resultado, las desigualdades de gasto entre los diferentes niveles formativos son superiores en los casos de limitación moderada o de ausencia de limitación que en el caso de limitación grave.

Esta mayor elasticidad del gasto evidencia una estrategia de adaptación a las circunstancias que hace que, cuando la discapacidad no supone una limitación grave, los hogares con menos recursos opten por reservar un importe sustancial de estos recursos a la adquisición de otros bienes esenciales, que pasan a ser prioritarios a la atención de la dimensión social de la discapacidad, mientras que los grandes gastos en la atención a la discapacidad solo se dan en caso de que la limitación sea grave y lo requiera. En cambio, los hogares con mayor nivel formativo tienen un comportamiento más estable respecto al gasto, lo que les permite seguir manteniendo unas condiciones de vida confortables, tanto para la persona discapacitada como para el resto de miembros del hogar, sea cual sea el grado de limitación asociado a la discapacidad, gracias al sostenimiento de unos niveles estructuralmente altos de gasto.

Aquí tenemos que señalar una única excepción, que queda recogida en el gráfico 2: hay un pico en el gasto de los hogares con estudios primarios y con limi- 
taciones graves, con un gasto cuyo valor medio es de 4.630 euros anuales. Esta excepción se explica porque en esta categoría se concentra la población de edad avanzada con limitaciones graves derivadas de la discapacidad, con unas rentas estables y con una menor proporción de gasto en otras partidas de primera necesidad (como, por ejemplo, la atención a niños, los costes de hipoteca o alquiler de la vivienda, que generalmente ya están amortizados), lo cual les permite dedicar una parte sustancial de sus recursos a la discapacidad. Si no se tiene en cuenta esta población de edad avanzada (aquí se ha realizado un segundo análisis limitado a la población de 16 a 64 años), la tendencia general descrita anteriormente, según la cual a mayor nivel formativo le corresponde un mayor gasto en discapacidad, gana nitidez.

\section{Gráfico 2. Gasto medio anual dedicado a la discapacidad, según el nivel de estudios de la persona de referencia y el grado de limitación de la persona discapacitada. España, 2008.}

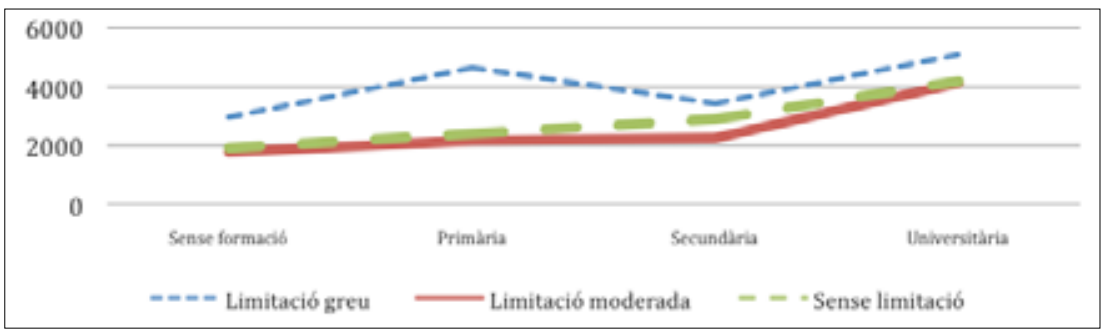

Fuente: elaboración propia a partir de la EDAD 2008.

En cuanto a la relación con el mercado de trabajo, los hogares donde la persona de referencia está trabajando pueden dedicar más gasto a la discapacidad, y este gasto es a la vez mayor si la ocupación es más cualificada (hipótesis 2.2). Así pues, el mayor gasto se da en los hogares donde la persona de referencia trabaja en el segmento superior del mercado de trabajo (directivo, gerente, técnico, científico, intelectual, de profesional o de técnico de apoyo, entre otros), con un intervalo del gasto que se mueve entre los 3.970 euros anuales si la persona discapacitada del hogar está moderadamente limitada, hasta los 5.374 euros si lo está gravemente. Tomando estos datos como referencia, los hogares donde la persona de referencia trabaja en un segmento inmediatamente inferior, como asalariado cualificado (administrativo, operario, vendedor, auxiliares, etc.), destinan un $40 \%$ menos a la discapacidad: entre 2.350 euros si la persona discapacitada está moderadamente limitada y 3.300 euros si lo está gravemente. La capacidad de dedicar recursos para atender la discapacidad se reduce todavía más en situaciones de 
paro o de ocupación no cualificada (mozos, peones, etc.), con un techo máximo de 2.000 euros anuales, sea cual sea el grado de limitación. Esto refleja de nuevo la influencia de la dimensión social de la discapacidad sobre el gasto de los hogares: los hogares con mejor posición en el mercado de trabajo pueden destinar el doble de recursos para atender la discapacidad que aquellos hogares que se concentran en las posiciones más precarias o que están excluidas del mundo laboral, incluso cuando en las primeras la limitación es moderada o inexistente y en las segundas es grave. Mención aparte merecen los hogares donde la persona de referencia está jubilada o incapacitada, ya que mayoritariamente se trata de hogares con personas de referencia de edad avanzada: aunque se encuentran fuera del mercado de trabajo, la relativa estabilidad en el ingreso de las pensiones les permite sostener y/o incluso aumentar el gasto destinado a la discapacidad con niveles similares a los de la población asalariada cualificada: cerca de 4.000 euros anuales en los casos de limitación grave y sobre los 2.000 euros en el resto de grados de limitación.

\section{Gráfico 3. Gasto medio anual dedicado a la discapacidad, según situación} de actividad de la persona de referencia y grado de limitación de la persona discapacitada. España, 2008.

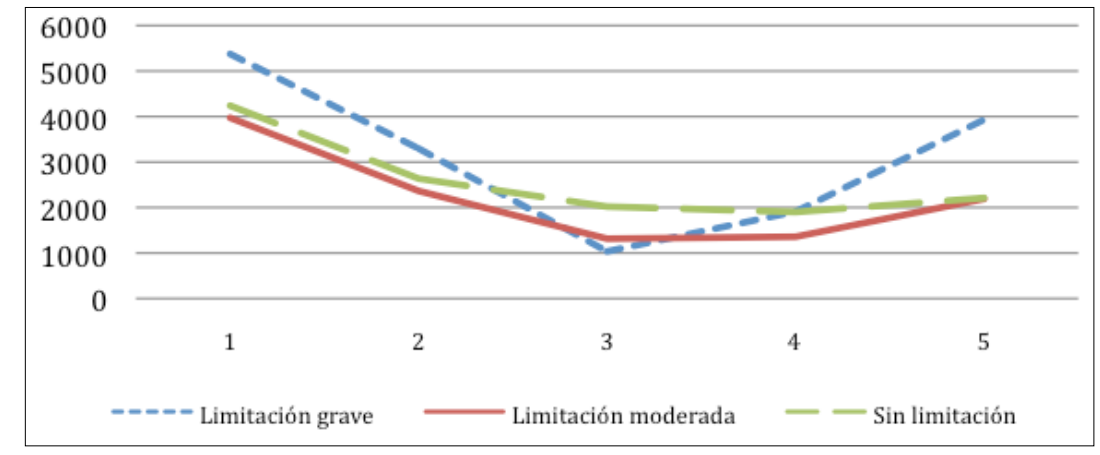

1 = Ocupado, dirección o técnico; 2 = Ocupado, asalariado cualificado; 3 = Ocupado, sin cualificación; 4 = Parado; 5 = Jubilado o Incapacitado.

Fuente: elaboración propia a partir de la EDAD 2008.

Además de estos dos indicadores tradicionales, hemos utilizado uno complementario para relacionar la posición social con el gasto dedicado a la discapacidad: la capacidad monetaria del hogar, medida en función de los ingresos mensuales de la persona de referencia. Mediante su uso pretendemos dar cuenta directamente de las posibilidades que tienen los hogares de adquirir bienes materiales y de su impacto en la adquisición de recursos para atender la discapacidad. 
Este es el indicador que presenta mayores diferencias de gasto en discapacidad, y sigue, como era de esperar, una relación positiva, según la cual cuanto más ingrese el hogar más gastará en atender la discapacidad (hipótesis 2.3). Los hogares donde la persona de referencia pertenece al tramo inferior de ingresos (inferiores a 1.000 euros mensuales) destinan una menor cuantía a la discapacidad, partiendo de 1.750 euros anuales y llegando a los 3.000 euros si la limitación es grave. A partir de estas cantidades, el aumento del gasto es progresivo y lineal, generalmente de un $30 \%$ a un $40 \%$ por cada tramo superior de ingresos (establecidos aquí en divisiones de 1.000 euros mensuales), de forma que las cantidades dedicadas a la discapacidad en los hogares pertenecientes al cuarto y último tramo (en que la persona de referencia ingresa más de 3.000 euros mensuales) duplican los de los hogares pertenecientes al primer tramo de ingresos, y se sitúan cerca de 7.000 euros anuales en casos de limitación grave, y cerca de 5.000 euros anuales en los otros niveles de limitación.

\section{Gráfico 4. Gasto medio anual dedicado a la discapacidad, según ingresos mensaules de la persona de referencia y grado de limitación de la persona discapacitada. España, 2008}

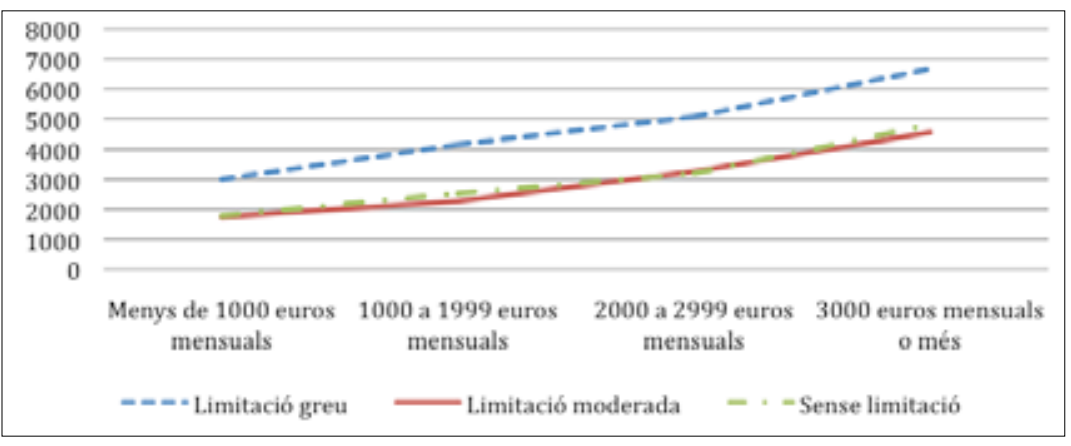

Fuente: elaboración propia a partir de la EDAD 2008.

Aunque hemos destacado la progresividad del gasto en discapacidad en relación con el tramo de ingresos al cual pertenece el hogar, hay que remarcar otra vez las diferencias que hay en el grado de progresividad, debidas al componente social de la discapacidad. En los casos en los que las personas con discapacidad del hogar están gravemente limitadas, el aumento del gasto entre los diferentes tramos de ingresos es sustancialmente menor (un 122\% entre el primer y el último tramo) que en los hogares donde la persona discapacitada no está limitada o lo está moderadamente (entre un $162 \%$ y un 170\%). Esto indica que, mientras que los hogares con menos posibilidades pueden reducir su aportación a la disca- 
pacidad cuando esta comporta una limitación moderada o nula, los hogares con mayores ingresos siguen manteniendo un gasto elevado, que no se destina tanto a la atención a la discapacidad médica sino que está pensado para mantener una mayor calidad de vida tanto de la persona con discapacidad como para el resto de personas que componen el hogar.

\section{El peso de la dimensión social en el gasto en discapacidad}

Llegados a este punto hemos buscado determinar qué parte del gasto total de los hogares puede atribuirse a la dimensión social de la discapacidad y qué parte a la simple dimensión físico-médica. Hemos agrupado las 3 variables analíticas (nivel de estudios, relación con la ocupación y cuantía mensual de ingresos) en una nueva dimensión explicativa, mediante un análisis de correspondencias múltiple. Esta dimensión, que puede ser considerada como la dimensión de posición social, es la que mayor varianza acumulaba respecto a las variables originales (con un autovalor de 1,973). Posteriormente, a través de un análisis de clasificación bietápica, hemos agrupado los hogares en categorías sociales homogéneas, es decir, similares entre sí y diferentes a los hogares del resto de grupos. Con este análisis se han obtenido 3 grupos de hogares diferenciados por su posición social, sobre los cuales hemos realizado un análisis comparativo del gasto en discapacidad.

Con esta finalidad hemos estimado un gasto medio de los hogares determinado por la dimensión físico-médica, y una variabilidad en torno a este gasto medio (por encima o por debajo) que se explica por la dimensión social. Los resultados indican que la dimensión social explica un $23,2 \%$ del gasto medio en discapacidad de los hogares españoles con personas con discapacidad, que es de 2.787 euros anuales. Además, como era de esperar, el peso de esta dimensión social es inversamente proporcional a la gravedad de la limitación de la discapacidad: representa el 15,3\% del gasto en los casos de limitación grave, el 23,7\% en los casos de limitación moderada y el 30,7\% en los casos de ausencia de limitación (hipótesis 3).

Como se aprecia en el gráfico 10, el gasto dedicado a la discapacidad es claramente superior en los hogares con limitación grave, especialmente debido al peso de la dimensión físico-médica (hipótesis 1), a la cual se destinan 3.145 euros de los 3.713 de media, frente a los 568 destinados a cubrir la dimensión social. En estos casos, la incapacidad severa de la discapacidad centra casi toda la atención de los recursos de los hogares, mientras que la dimensión social queda al alcance solo de los hogares con una mejor posición social. En cambio, si la limitación pro- 
ducida por la discapacidad no es grave, el gasto medio se reduce sustancialmente, sobre todo debido a la reducción del gasto explicado por la dimensión físicomédica, a la cual se dedican menos de 2.000 euros anuales de media, mientras que la dimensión social se mantiene en unos valores absolutos estables, entre 516 y 836 euros anuales, debido al esfuerzo que los hogares más acomodados siguen realizando para atender la discapacidad, en términos sociales de mejora de las condicionas de vida.

\section{Gráfico 5. Gasto medio anual dedicado a la discapacidad, según estructura del gasto. España, 2008}

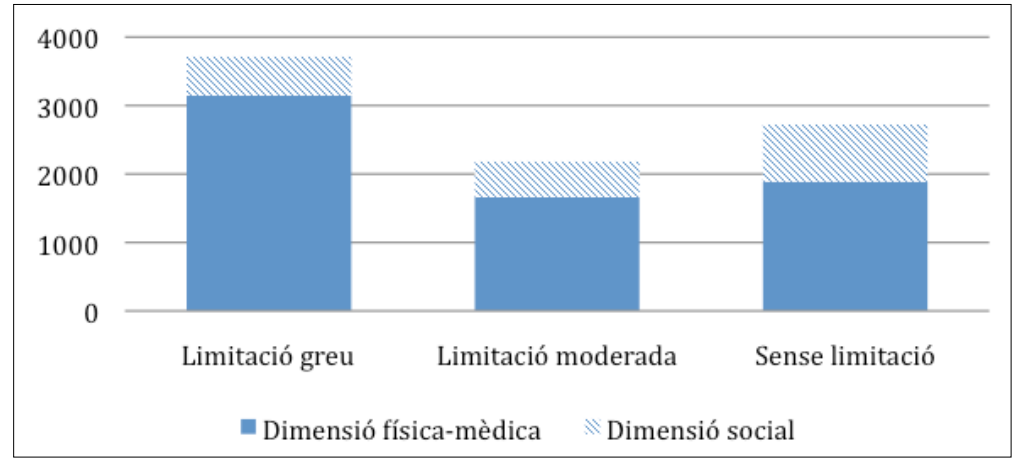

Fuente: elaboración propia a partir de la EDAD 2008.

\section{Conclusiones}

Hemos analizado los datos relativos al gasto en discapacidad, que ponen de relieve que la atención a la dimensión social de la discapacidad hace que los hogares con más recursos inviertan una mayor cuantía para atender la discapacidad, mientras que los hogares con menos recursos invierten una cantidad claramente inferior. Mediante el análisis de los diferentes gastos familiares hemos podido cuantificar el efecto de esta dimensión social. De hecho, a la hora de evaluar las estrategias de los hogares para hacer frente a la discapacidad encontramos que, en algunas ocasiones, esta dimensión social adquiere un mayor peso que la dimensión físicomédica. Así, a igual grado de limitación, los hogares con rentas superiores a 3.000 euros mensuales dedican una cantidad media a atender la discapacidad entre un $120 \%$ y un $170 \%$ superior que aquellos hogares con una renta inferior a 1.000 euros mensuales. Menos previsible es que, incluso entre diferentes niveles de gravedad, las diferencias entre hogares sigan siendo sustanciales. Así, solo aceptando la importancia de la dimensión social de la discapacidad podemos entender que los hogares acomodados donde el miembro con discapacidad no tiene ninguna 
limitación sigan invirtiendo entre un 40\% y un 60\% más al atender la discapacidad que los hogares con menos recursos en los que la persona discapacitada está gravemente limitada.

Es por esto que entendemos que la atención a la discapacidad presenta un núcleo irreducible de necesidades que todos los hogares con miembros con discapacidad tienen que afrontar, sea cual sea su posición económica. Superado este núcleo inicial entra en escena la dimensión social de la discapacidad, que se caracteriza por un comportamiento más elástico: los hogares con más recursos tienden a aumentar el gasto para ampliar y mejorar la atención a sus miembros con discapacidad, sin un horizonte máximo nítido, con el que consiguen mejorar las condicionas de vida tanto de las personas con discapacidad cómo del resto de miembros del hogar. Bajo la óptica de estos hogares, el gasto en discapacidad no es solo paliativo sino que representa, además, una inversión para el mantenimiento de la posición social del hogar. Por otro lado, los hogares situados en posiciones socioeconómicas menos ventajosas tienen más dificultades para dar respuesta a esta dimensión social, y la vulnerabilidad inherente a su posición se agrava por la presencia de individuos con discapacidad, que, además de limitar la entrada de rentas al hogar (por sus mayores dificultades de inserción laboral), también ejercen presión sobre la proporción de recursos disponibles del hogar dedicados a su atención.

En definitiva, la dimensión social de la discapacidad se caracteriza por su elasticidad. Cuando la discapacidad es grave se acortan las diferencias entre posiciones sociales desiguales: todos los grupos sociales tienen que invertir en la discapacidad, sin excusas, independientemente del poder adquisitivo. Las diferencias de gasto entre diferentes grupos de hogares no son estadísticamente significativas o, en todo caso, únicamente en los hogares donde la persona de referencia se sitúa en los escalones superiores de la jerarquía social, donde se invierte una cuantía significativamente superior al resto en atención a la discapacidad. En cambio, cuando la persona discapacitada puede ejercer actividades cotidianas sin una limitación grave, las diferencias entre los diferentes grupos sociales aumentan por el hecho de que, en estos casos, la dimensión social adquiere una mayor presencia: es la porción de gastos que no forma parte del núcleo irreducible de la discapacidad la que incide en la diferenciación social. 


\section{Bibliografía}

Albert, B. (ed.) (2005). In or Out of the Mainstream?: Lessons from research on disability and development cooperation. Leeds: The Disability Press.

Amiralian, M. L. et alii (2000). «Conceituando deficiência». Revista de Saúde Pública, 34 (1): 97-103.

Banco Mundial (BM) (2007). Social Analysis and Disability: A Guidance Note. Nueva York: Banco Mundial.Última consulta 15/11/2013:<http://siteresources.worldbank.org/DISABILITY/Resources/280658-1172606907476/ SAnalysisDis.doc. >

Barnes, C. (2010). «Discapacidad, política y pobreza en el contexto del "mundo mayoritario"». Politica y Sociedad, 47 (1): 11-25.

- y Mercer, G. (eds.) (2005). The Social Model of Disability - Europe and the Majority World. Leeds: The Disability Press.

Barrientos, A.; Gorman, M. y Heslop, A. (2003). «Old age poverty in developing countries: Contributions and dependence in later life». World Development, 31 (3): 555-570.

Braña, F. J. y Antón, J. I. (2011). «Pobreza, discapacidad y dependencia en España». Papeles de Economía Española, 129: 14-25.

Cantanero, D.; Moreno, P. (2011), «Dependencia, discapacidad y temporalidad en el empleo». Papeles de Economía Española, 129: 98-109.

Cantó, O.; Del Río, C. y Gradín, C. (2000). «La situación de los estudios de desigualdad y pobreza en España». Cuadernos de Gobierno y Administración, 2: 25-94.

Chapireau, F. (2001). «Mort ou renaissance d'une classification», en Classification Internationale des handicaps et santé mentale. Paris: Éditions CTNERHIGFEP: $21-51$.

Colectivo IOÉ (2003). La inserción laboral de las personas con discapacidades. Barcelona: Fundación La Caixa.

- (2013). «Discapacidad y dependencia en España. Diagnóstico de las personas con diversidad funcional», Papeles de Relaciones Ecosociales y Cambio Global, 120: 129-139.

De la CaL, M. L. (2009). «Avances metodológicos para el análisis y la comprensión de la desventaja social femenina». Revista Internacional de Organizaciones, 3: 29-47.

Díaz, E. (2011). «Estratificación y desigualdad por motivo de discapacidad». Intersticios, 5 (1): 157-170.

- (2009). «Reflexiones epistemológicas para una sociología de la discapacidad». Intersticios, 3 (2): 85-99. 
Dobson, B.; Middleton, S. y Beardsworth, A. (2001). The impact of childhood disability on family life. York: Joseph Rowntree Foundation.

Egea, C. y Sarabia, A. (2001). «Clasificaciones de la OMS sobre discapacidad». Boletín del Real Patronato sobre Discapacidad, 50: 15-30.

- (2004). «Visión y modelos conceptuales de la discapacidad». Polibea, 73: 1-20.

Ferreira, M. A. y Díaz, E. (2007). «La discapacidad: una modalidad inexplorada de exclusión social». III Congreso Nacional Discapacidad y Universidad. Madrid. Última consulta 18/11/2013: <http //www.um.es/discatif/TEORIA/FerreiraDiaz_Zaragoza.pdf.>

García-Gómez, P; Hernández-Quevedo, C.; López-Nicolás, A. (2008). «Renta absoluta y renta relativa: ¿cuál es su papel en la determinación de las desigualdades en salud?», en Rodríguez, M. y Urbanos, R. (eds.), Desigualdades sociales en salud. Factores determinantes y elementos para la acción. Madrid: Elsevier Masson.

Gerschicк, T. J. (2000). «Toward a theory of disability and gender». Signs, 25 (4): 1263-1268.

Gordon, D. (2005). Indicators of Poverty E Hunger, en Expert Group meeting on youth development indicators. University of Bristol. Última consulta 15/11/2013: <http://students.oikonomika.org/sociology/sources/gordon_ poverty.pdf.>

Gustavsson, A. (2004). «The role of theory in disability research-springboard or strait-jacket?». Scandinavian Journal of Disability Research, 6 (1): 55-70.

Haveman, R. y Bershadker, A. (2001). "The 'Inability to be Self-Reliant' as an indicator of Poverty: Trens for the US, 1975-97». Review of Income and Wealth, 47 (3):335-360.

Hemmingsson, H. y Jonsson, H. (2005). «An occupational perspective on the concept of participation in the International Classification of Functioning. Disability and Health-some critical remarks». The American Journal of Occupational Therapy, 59 (5): 569-576.

Huete, A.; Sola, A. y Lara, P. (2010). Los jóvenes con discapacidad en España. Informe de situación 2010. Madrid: Cinca.

Hughes, B. y Paterson, K. (1997). «The social model of disability and the disappearing body: Towards a sociology of impairment». Disability E Society, $12(3): 325-340$.

INE (2010). Encuesta de Discapacidades, Autonomía y Dependencia: metodología. Online: INE.

Kidd, M.; Sloane, P. y Ferko, I. (2000). «Disability and the labour market: an analysis of Brithis males». Journal of Health Economics, 19 (6): 961-981. 
Lustig, D. C. y Strauser, D. R. (2007). «Causal relationships between poverty and disability». Rehabilitation Counselling Bulletin, 50 (4): 194-202.

Malo, M. A. y Dávila, D. (2006). La protección social de las personas con discapacidad: ayudas técnicas, ayudas personales y pobreza. Madrid: FIPROS/Seguridad Social. Última consulta: 12/04/2013: <http://www.seg-social.es/ prdi00/groups/public/documents/binario/51593.pdf.>

Mercader-Prats, M. (1998). «Identifying Low Standards of Living: Evidence from Spain». Research on Economic Inequality, 8: 155-173.

Michailakis, D. (2003). «The Systems Theory Concept of Disability: one is not born a disabled person, one is observed to be one». Disability $\mathcal{E}$ Society, 18 (2): 209-229.

Minkler, M.; Fuller-Thomson, E. y Guralnik, J. M. (2006). «Gradient of disability across the socioeconomic spectrum in the United States». New England Journal of Medicine, 355 (7): 695-703.

Newacheck, P. W.; Inkelas, M. y Kim, S. E. (2004). «Health services use and Health care expenditures for children with disabilities», Pediatrics, 114 (1): $79-85$.

OMS (Organización Mundial de la Salud) (2001). Clasificación Internacional del Funcionamiento, de la Discapacidad y de la Salud. Ginebra: Organización Mundial de la Salud.

ONU Enable (2008). Factsheet on Persons with Disabilities. Nueva York: United Nations. Última consulta: 15/11/2013: <http://www.un.org/disabilities/ default.asp?navid $=348$ pid $=18>$.

Pereda, C.; De Prada, M. A. y Actis, W. (2012). Discapacidades e inclusión social. Barcelona: Obra Social «La Caixa».

Pérez, S. (2009). «El estudio de la pobreza en España desde una óptica económica: medición y políticas». Estudios de Economía Aplicada, 27 (2), 249-272.

Regidor, E. (2008). «Desigualdades socioeconómicas en la exposición al riesgo y en salud», en M. Rodríguez y R. Urbanos, (eds.), Desigualdades sociales en salud. Factores determinantes y elementos para la acción. Madrid: Elsevier Masson.

Regidor, E.; Ronda, E.; Pascual, C;; Martínez, D.; Calle, M. y DomínGUEZ, V. (2006). «Decreasing socioeconòmic inequalities and increasing Health inequalities in Spain: A case study», American Journal of Public Health, 96 (1): 102-108.

Rodríguez, M. y Urbanos, R. (eds.) (2008). Desigualdades sociales en salud. Factores determinantes y elementos para la acción. Madrid: Elsevier Masson. 
Rodríguez-Sanz, M. y Carrillo, P.; Borrell, C. (2006). Desigualdades sociales en la salud, los estilos de vida y la utilización de servicios sanitarios en las CCAA., 1993-2003. Madrid: Observatorio de Salud de la Mujer, Ministerio de Sanidad y Consumo.

Rossignol C. (1999). Inadaptation, Handicap, Invalidation? Histoire et étude critique des notions, de la terminologie et des pratiques dans le champ professionnel de l'Éducation spéciale. Tesis doctoral, Université Louis Pasteur. Lille: Éditions du Septentrion.

- (2006). «Altérations corporelles, dysfonctionnements et handicaps. Pour une classification de l'usage des concepts». Le Furet, 49: 14-16.

Sen, A. (1984). Poverty and Famines: an essay on entitlement and deprivation. Nueva York: Oxford University Press.

- (1995). Nuevo examen de la desigualdad. Madrid: Alianza Editorial.

- (1999). Desarrollo y libertad. Madrid: Planeta.

Vehmas, S.y Taylor, S. (2004). «Ethical Analysis of the Concept of Disability». Mental Retardation, 42 (3): 209-222.

Verdugo, M. A. (2003). «La concepción de la discapacidad en los modelos sociales» en Francisco de BorJa Jordán de UrRíes (coord.), Investigación, innovación y cambio: $V$ Jornadas Cientificas de Investigación sobre personas con discapacidad. Salamanca: Amarú: 235-247. 